\title{
Supporting Information \\ Disentangling the Contribution of Each Descriptive Characteristic of Every Single Mutation to Its Functional Effects
}

\author{
C. K. Sruthi ${ }^{1}$, Meher K. Prakash ${ }^{1, *}$ \\ ${ }^{1}$ Theoretical Sciences Unit, \\ Jawaharlal Nehru Centre for Advanced Scientific Research, \\ Bangalore-560064, India \\ *Corresponding author : meher@jncasr.ac.in
}




\section{Figures}

A

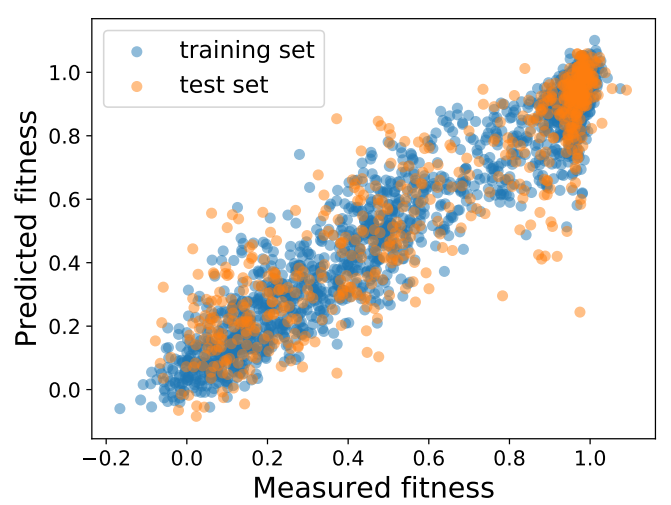

B

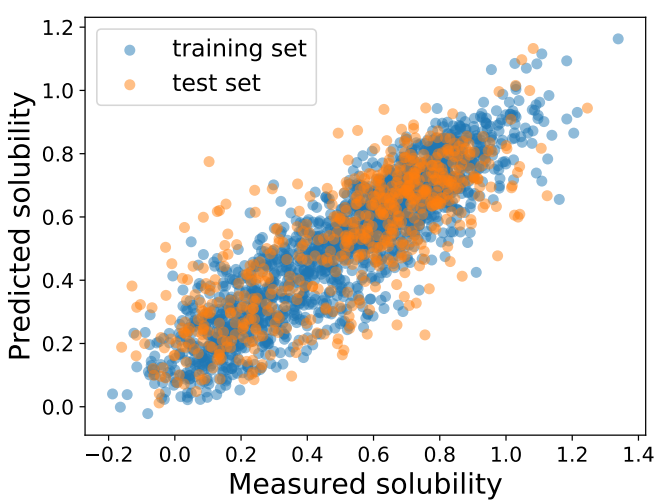

Figure S1. Quality of predictions. A comparison of the predicted mutational effect scores with the observed scores from the deep mutational scan of $\beta$-lactamase for (A.) fitness and (B.) solubility changes. The predictions were in good agreement with the observations. $75 \%$ of the data was used for training.
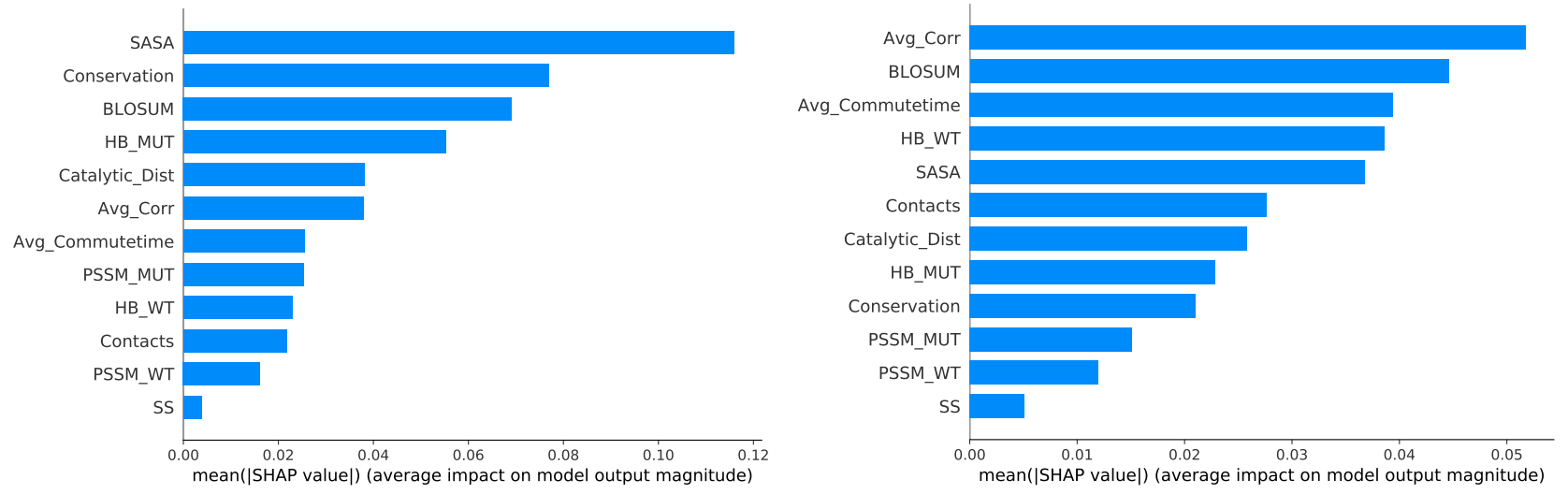

Figure S2. Overall importance of features. The average contribution of each variable towards prediction as quantified using average of absolute SHAP values is shown for the fitness (left) and solubility (right) data sets of $\beta$-lactamase. This figure reporting mean values is a summary of the Figure 2 in the article, where the data from all mutations is presented. 


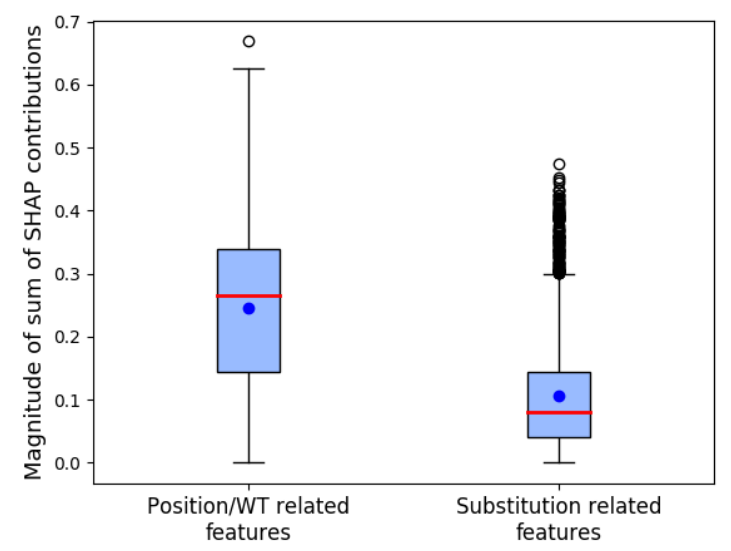

Figure S3. Relative contributions of groups of variables. The relative contributions of the descriptive variables which mainly reflect the wild-type context are compared with the contributions from the descriptors which describe the precise nature of the mutation. The absolute values of the SHAP contributions from these two groups are shown for the fitness prediction of variants of $\beta$ lactamase. It is clear that more significant contributions arise from the wild-type context, rather than the precise mutations. An intuitive example is that a significant fraction of mutational effects can be understood from the knowledge of solvent accessibility or conservation of the amino acid being replaced. The blue filled circle, red line and black open circles represent the mean, median and the outliers of the distribution. The lower and upper whiskers are plotted at the lowest data point greater than $Q_{1}-1.5 \times\left(Q_{3}-Q_{1}\right)$ and the greatest data point less than $Q_{3}+1.5 \times\left(Q_{3}-Q_{1}\right)$ respectively, where $\left(Q_{3}-Q_{1}\right)$ is the interquartile range shown using the blue box. $Q_{3}$ and $Q_{1}$ are the third and first quartiles respectively. 
$\mathbf{A}$
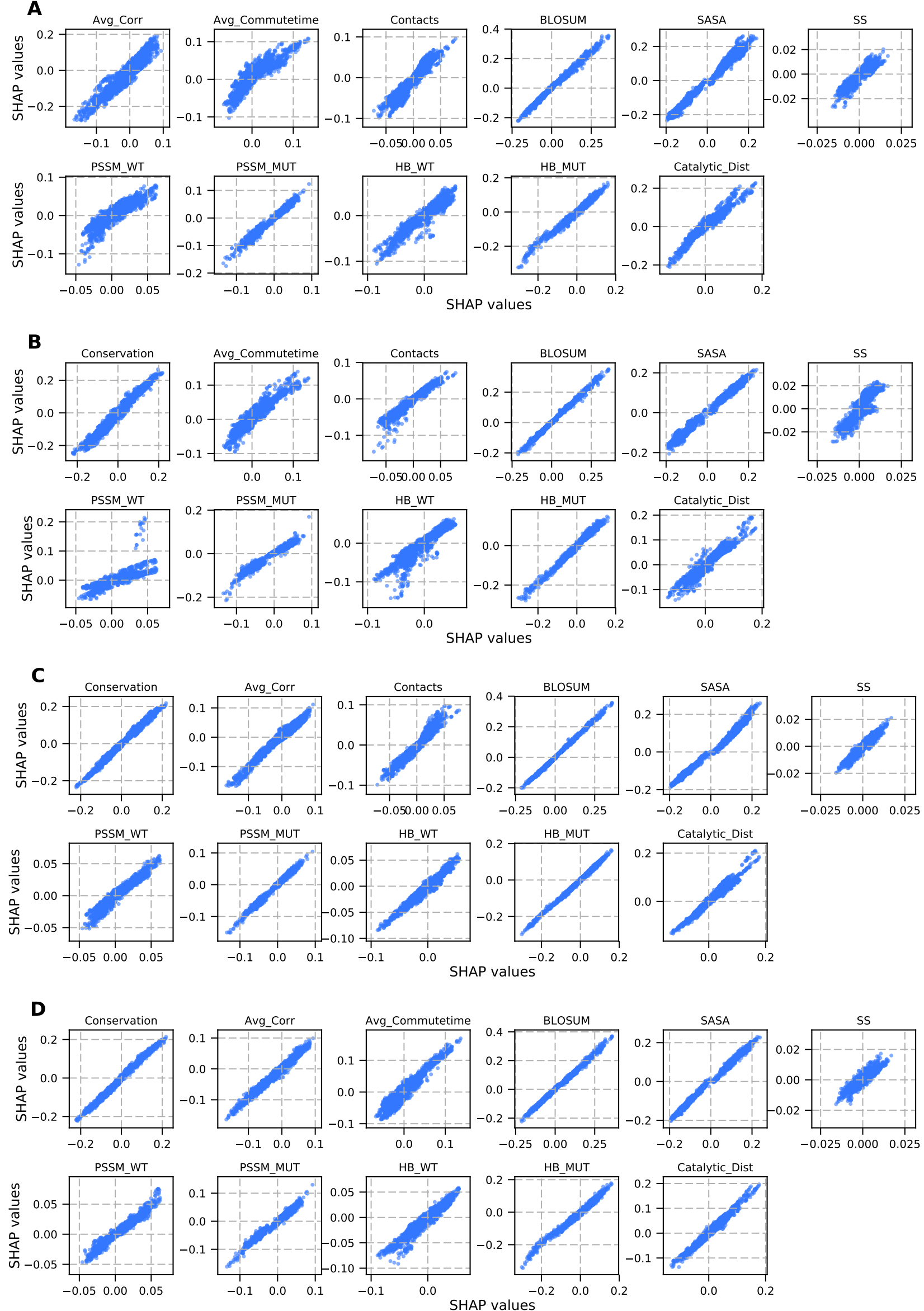

Figure S4. Robustness against removal of a single feature. Comparison of SHAP values from the model with all features (X-axis) with that from the reduced models where a single feature (A.) Conservation (B.) Avg_Corr (C.) Avg_Commutetime (D.) Contacts is removed (Y-axis). More reduced models are presented in Figures S5 and S6. 
A
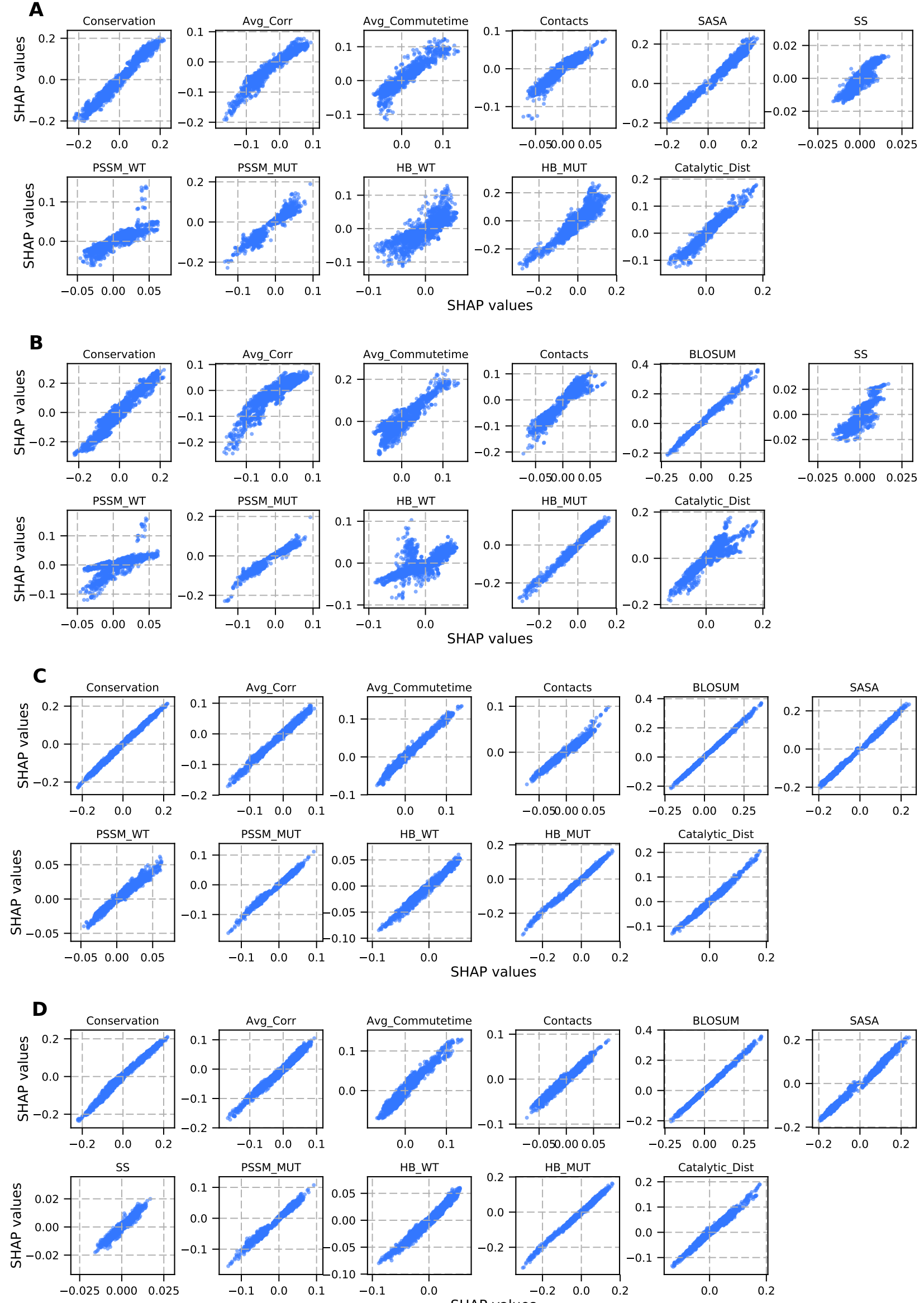

Figure S5. Robustness against removal of a single feature. Comparison of SHAP values from the baseline model in which all features were present with that from the reduced models where a single feature (A.) BLOSUM (B.) SASA (C.) SS (D.) PSSM_WT is removed. More reduced models are presented in Figures S4 and S6. 
A
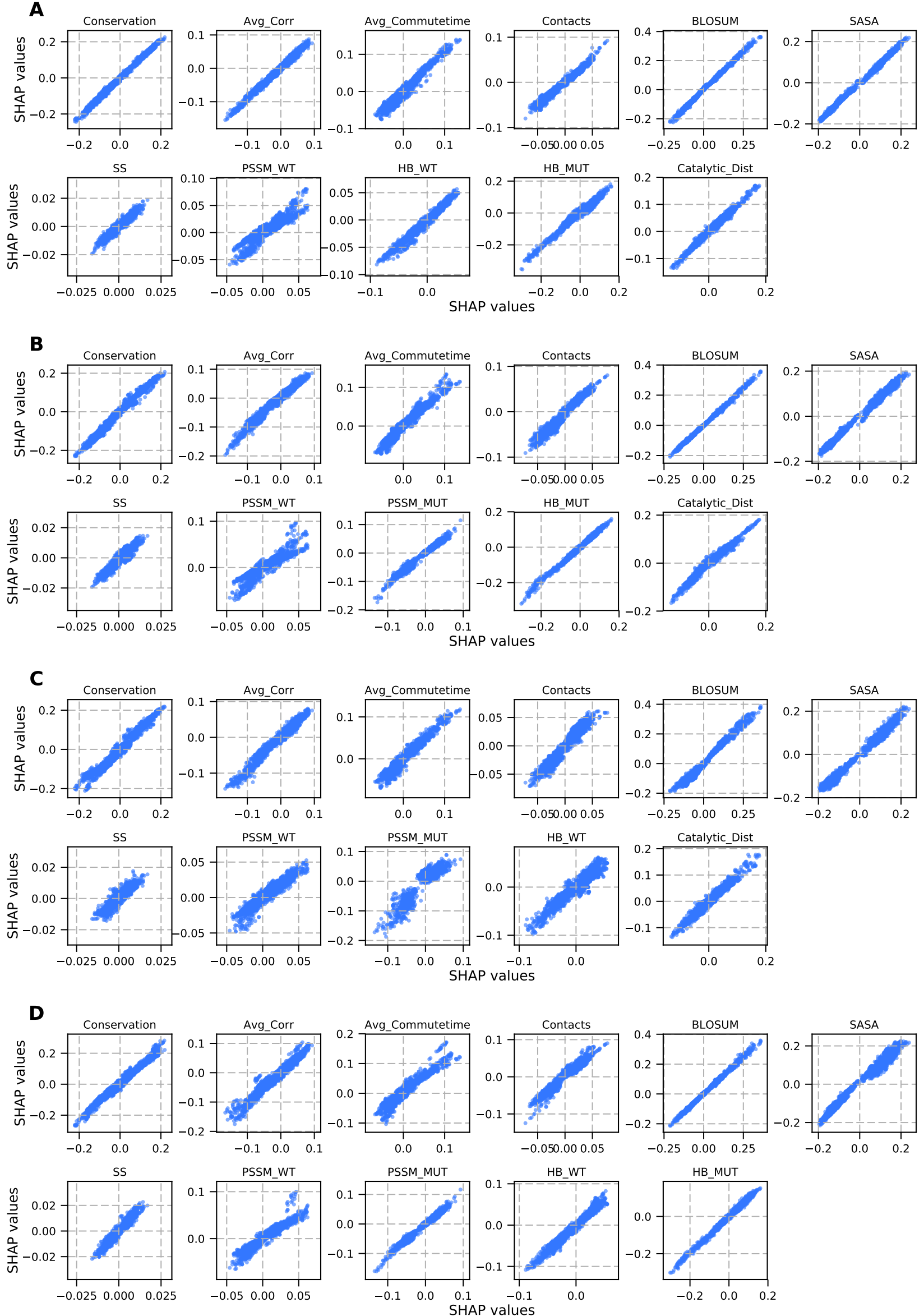

Figure S6. Robustness against removal of a single feature. Comparison of SHAP values from the model in which all features were present (X-axis) with that from the reduced models where a single feature (A.) PSSM_MUT (B.) HB_WT (C.) HB_MUT and (D.) Catalytic_Dist is removed (Y-axis). More reduced models were presented in Figures S4 and S5. 




Figure S7. Summary of comparisons between the models with fewer features and that with all features. The distribution of Spearman correlations between the SHAP values when one feature was removed from the model at a time and the model in which all features were used, from all the subplots in Figures S4, S5 and S6. 


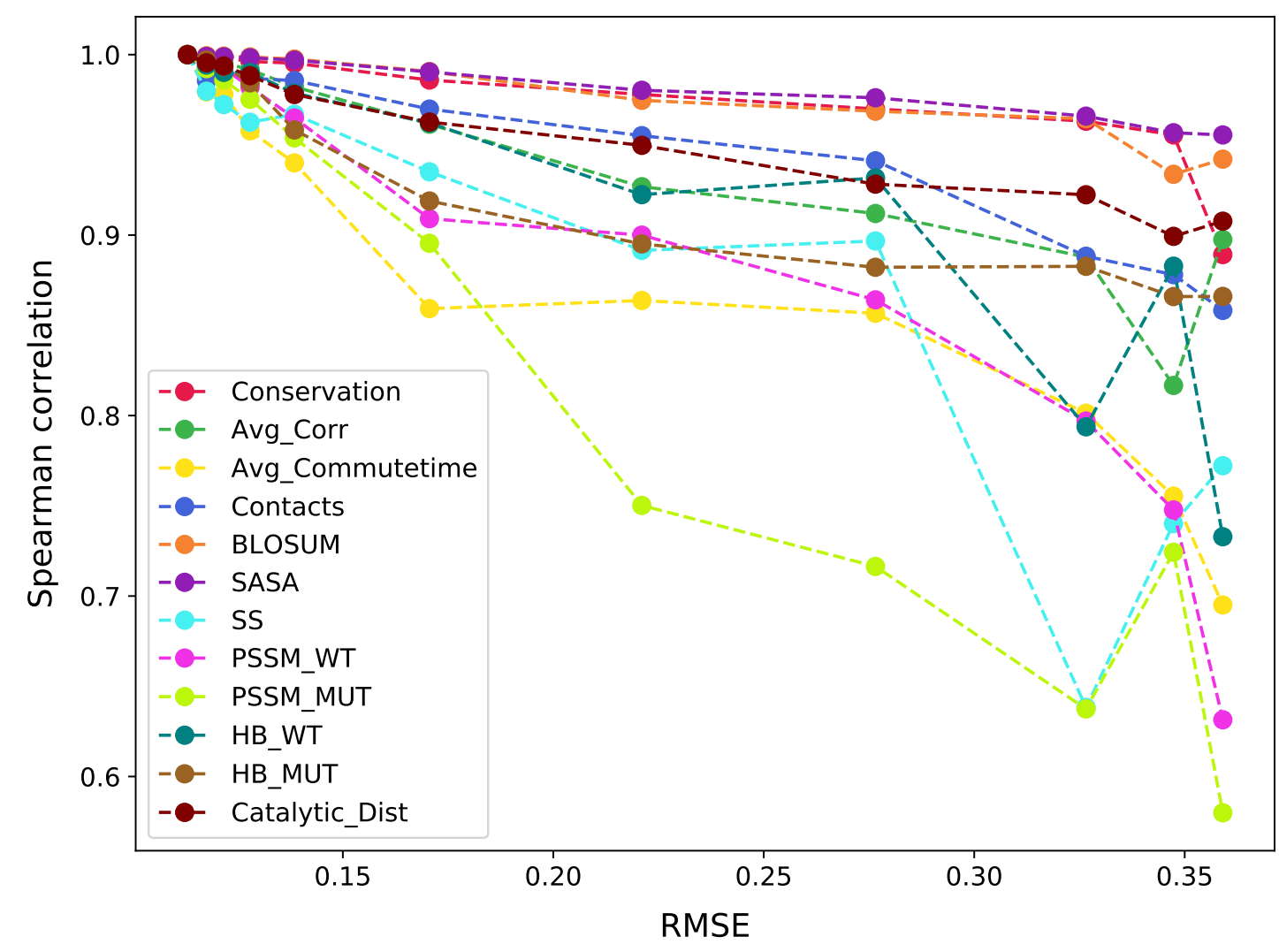

Figure S8. Robustness of SHAP values against reducing model accuracy Models with reduced predictive power were generated for the fitness data of $\beta$-lactamase as described in the article, by systematically reducing the $n$ _estimators parameter. The correlation between SHAP values corresponding to each feature from the less accurate models and that from the model with the highest predictive ability are calculated. The correlation is quantified using the Spearman correlation (Y-axis) and the model accuracy using the RMSE between the predictions and the experimental observations (Y-axis). 

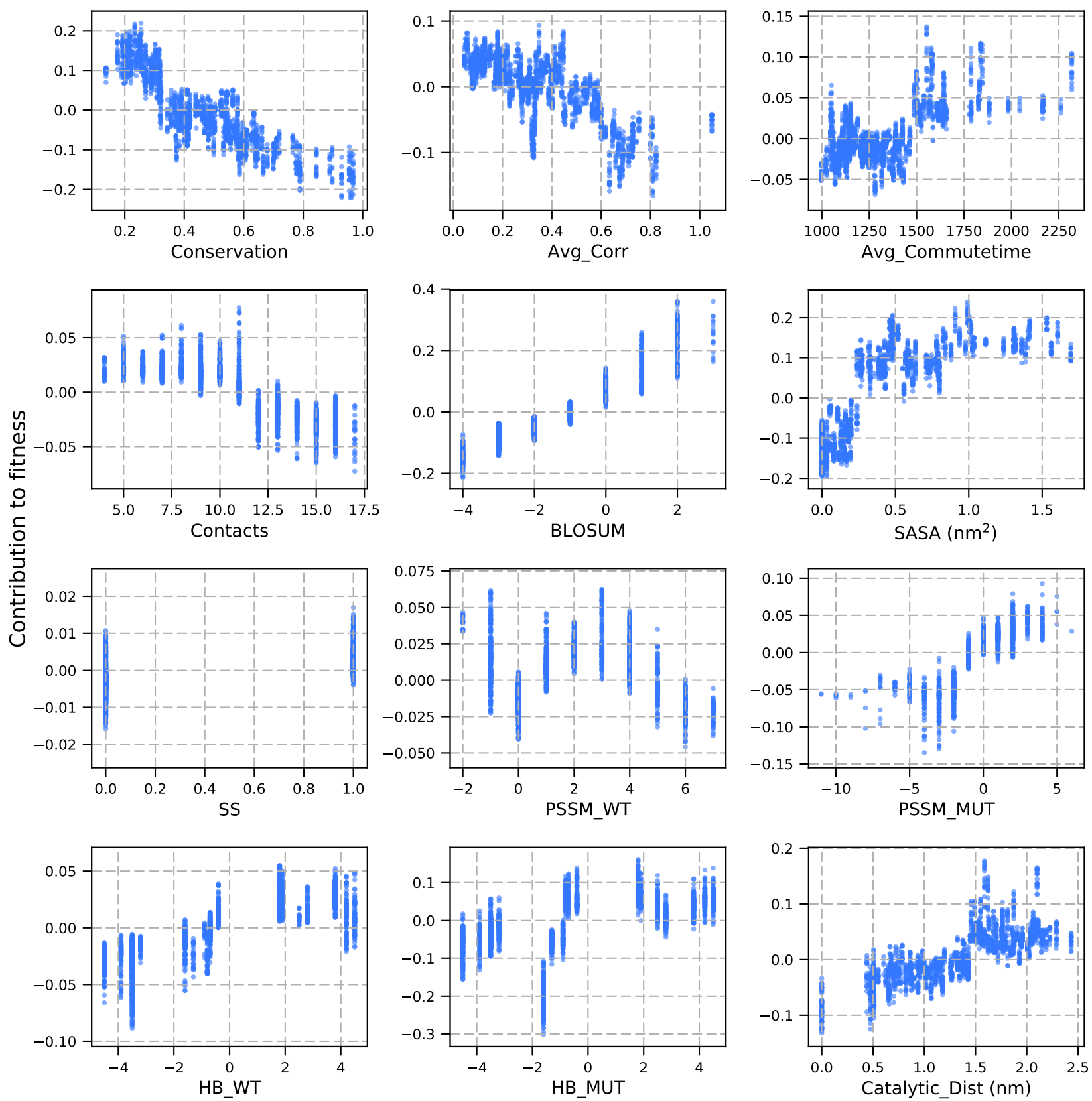

Figure S9. Feature contribution to fitness. The SHAP contributions of each feature towards the fitness predictions of $\beta$-lactamase are plotted with the corresponding feature values. The graphs of results relative to conservation and distance from the catalytic site, already presented in the Figure 3 of the article, are shown again for the completeness of the analysis. 

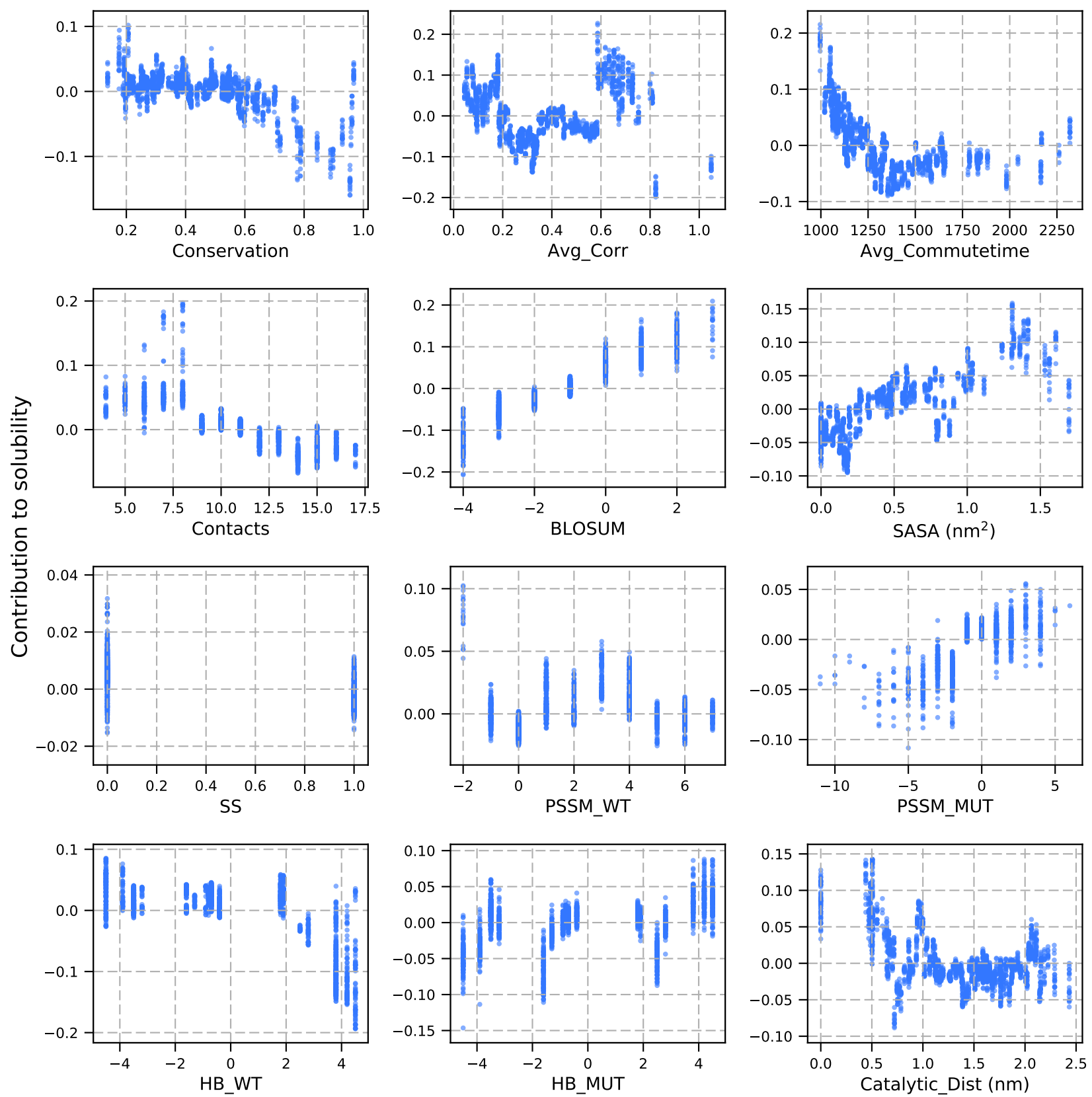

Figure S10. Feature contribution to solubility. The SHAP contribution of each feature to the prediction of mutational effects on solubility of $\beta$-lactamase are shown with respect to the feature values. The plots relative to conservation and distance from the catalytic site which are included in the Figure 3 of the article, are shown again for the completeness of the analysis. 




Figure S11. Role of number of wild-type contacts. For the 8 proteins we analyzed, the contribution from the number of contacts a wild-type amino acid has with its neighboring amino acids, to the overall fitness changes observed in a mutational scan is shown.
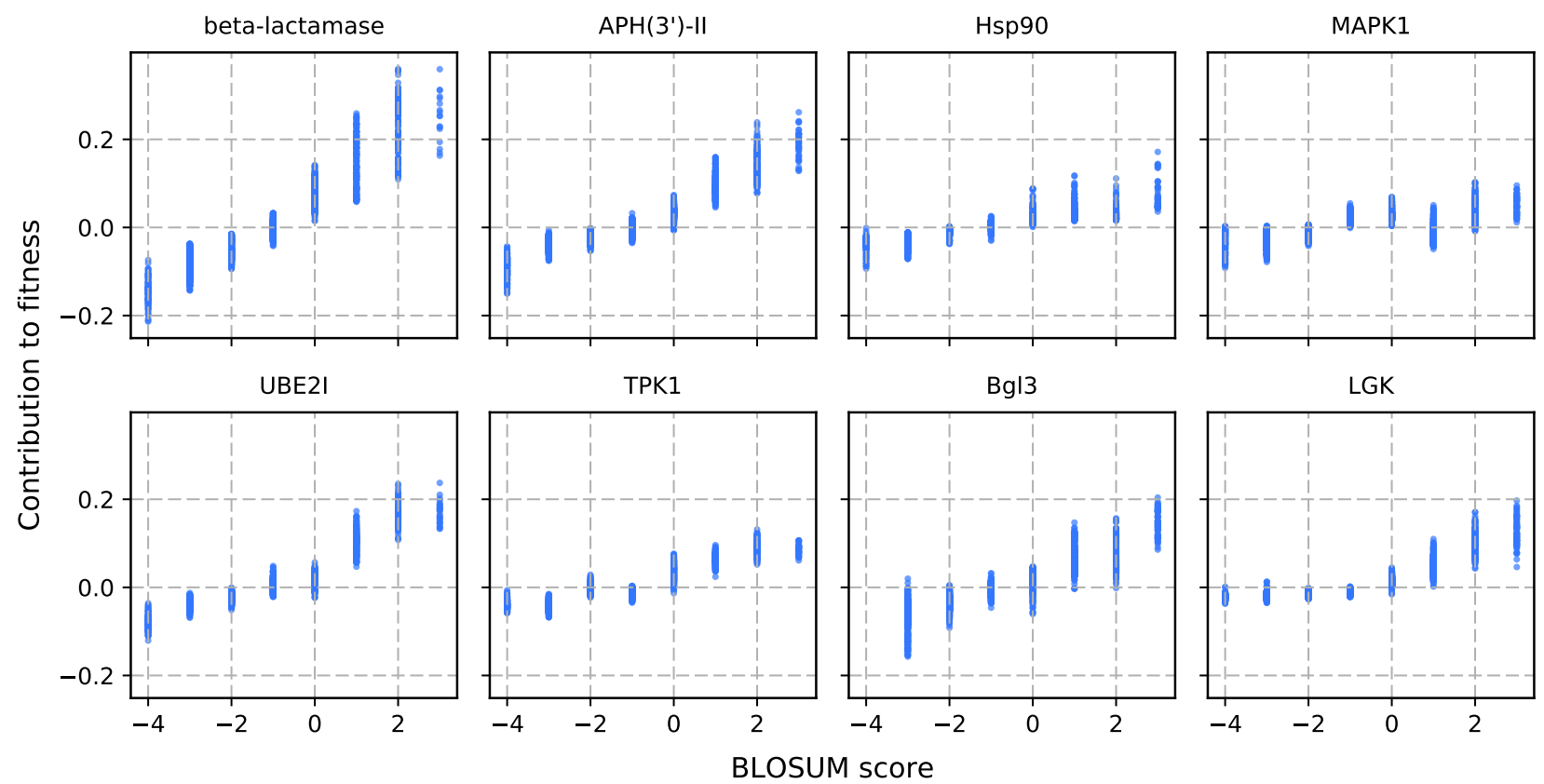

Figure S12. Role of substitution matrix score. The substitution effects are generally described using BLOSUM substitution matrix scores. The analysis shows the degree of contribution of this descriptive feature to the overall mutational effects. 




Figure S13. Role of solvent accessibility. The contribution of solvent accessible surface area(SASA) to the mutational effects is shown for the eight proteins. 


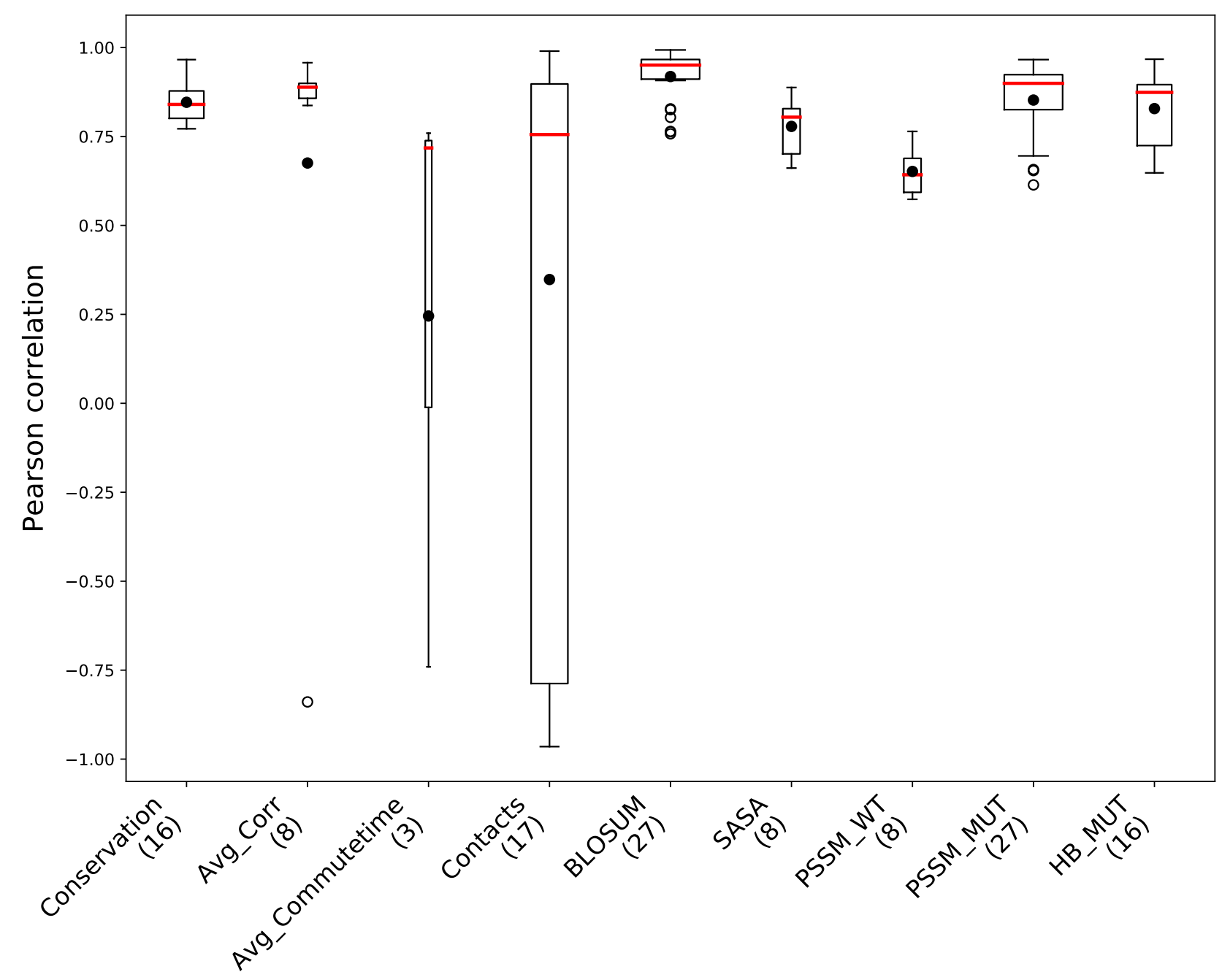

Figure S14. Quantifying similarity in trends across proteins. The trends of SHAP values across proteins for each feature was analyzed by binning the feature and calculating the mean SHAP values for each bin, and quantifying the correlation between these mean SHAP values across protein pairs. The distribution of the correlations which are significant ( $\mathrm{p}$-value $<0.05$ ) are shown for each feature. The black dot and the red line represent the mean and the median of the correlations respectively. The feature HB_WT did not have any correlations that are significant and hence not shown. Also the feature SS is not shown, as it takes only two feature values, 0 and 1 , and calculating correlation when there are only two data points is not meaningful. The width of the box is set proportional to the number of significant correlations, represented in parenthesis along with the x-labels. 


\section{Tables}

Table S1. Deep mutational scanning data sets used in the analysis.

\begin{tabular}{cccc}
\hline Protein & $\begin{array}{c}\text { No. of variants } \\
\text { included in the } \\
\text { analysis }\end{array}$ & $\begin{array}{c}\text { Structure } \\
\text { (PDB ID) }\end{array}$ & Reference \\
\hline $\begin{array}{c}\text { 3-lactamase } \\
\text { APH(3')-II }\end{array}$ & 4234 & $1 \mathrm{M} 40$ & Stiffler et al. $^{1}$ \\
Hsp90 & 4021 & 1ND4 & Melnikov et al. $^{2}$ \\
MAPK1 & 4470 & $4 \mathrm{CG} 9$ & Mishra et al. $^{3}$ \\
UBE2I & 2563 & $2 \mathrm{UYZ}$ & Brenan et al. $^{4}$ \\
TPK1 & 3181 & $3 \mathrm{~S} 4 \mathrm{Y}$ & Weile et al. $^{5}$ \\
Bg13 & 2732 & Weile et al. & \\
LGK & 6155 & 4ZLU & Romero et al. $^{6}$ \\
\hline
\end{tabular}

Table S2. The steps in the hyperparameter optimization for the AI model. The values that were evaluated based on model predictive ability through a 5-fold cross-validation approach for each parameter are also given.

\begin{tabular}{c|c|c}
\hline Step No. & Hyperparameters tuned & Parameter values sampled \\
\hline 1 & $\begin{array}{c}\text { max_depth, the maximum depth } \\
\text { possible for each tree in the ensemble } \\
\text { of trees } \\
\text { min_child_weight, the minimum } \\
\text { number of samples that a node has in } \\
\text { the tree }\end{array}$ & $3,5,7,9,15,20,25,50$ \\
\hline \multirow{2}{*}{3} & $\begin{array}{c}\text { gamma, minimum loss reduction } \\
\text { required for further division of a node } \\
\text { of a tree }\end{array}$ & $0,0.1,0.2,0.3,0.4,0.5,0.6,0.7,0.8,7,9,15,20,25,50$ \\
& $\begin{array}{c}\text { subsample, fraction of total training } \\
\text { samples that has to be used for } \\
\text { growing a tree at each iteration } \\
\text { colsample_bytree, fraction of total } \\
\text { features used at each iteration of } \\
\text { training }\end{array}$ & 0.9 \\
\hline 4 & $\begin{array}{c}\text { reg_alpha, weight for the L1 } \\
\text { regularization term }\end{array}$ & $0,0.0001,0.001,0.01,0.1,1,10,100$ \\
\hline
\end{tabular}


Table S3. The optimized set of hyperparameters for the different proteins are shown. Also shown are the quality of the results described by the root mean squared error (RMSE) and the Pearson-correlation $(\rho)$ for the training and test sets.

\begin{tabular}{|c|c|c|c|c|c|c|c|c|c|c|c|}
\hline Protein & n_estimators & max_depth & min_child_weight & gamma & subsample & colsample_bytree & alpha & $\mathbf{R M S E}_{\text {training }}$ & $\mathbf{R M S E}_{t e s t}$ & $\rho_{\text {training }}$ & $\rho_{\text {test }}$ \\
\hline $\begin{array}{c}\beta \text {-lactamase } \\
\text { (solubility) }\end{array}$ & 1116 & 5 & 3 & 0 & 0.8 & 0.7 & 0.0001 & 0.12 & 0.18 & 0.9 & 0.79 \\
\hline $\begin{array}{c}\beta \text {-lactamase } \\
\text { (fitness) }\end{array}$ & 1247 & 9 & 25 & 0 & 0.8 & 0.8 & 0 & 0.1 & 0.15 & 0.96 & 0.91 \\
\hline APH(3')-II & 1253 & 7 & 15 & 0 & 0.8 & 0.4 & 0.01 & 0.14 & 0.2 & 0.89 & 0.77 \\
\hline Hsp90 & 1218 & 5 & 1 & 0 & 0.6 & 0.6 & 0 & 0.1 & 0.14 & 0.93 & 0.86 \\
\hline MAPK1 & 1550 & 7 & 25 & 0 & 0.7 & 0.4 & 0.001 & 0.14 & 0.18 & 0.88 & 0.79 \\
\hline UBE2I & 918 & 7 & 20 & 0 & 0.9 & 0.4 & 0.01 & 0.17 & 0.21 & 0.85 & 0.72 \\
\hline TPK1 & 276 & 7 & 7 & 0 & 0.7 & 0.4 & 0.001 & 0.23 & 0.27 & 0.68 & 0.34 \\
\hline Bgl3 & 660 & 7 & 5 & 0 & 0.9 & 0.7 & 0.001 & 0.11 & 0.18 & 0.92 & 0.75 \\
\hline LGK & 804 & 7 & 7 & 0.1 & 0.7 & 0.4 & 0 & 0.18 & 0.2 & 0.69 & 0.52 \\
\hline
\end{tabular}

Table S4. Comparison of quality of predictions with studies where protein-specific models were developed training on a subset of variants from deep mutational scan data. The Pearson correlation reported for the test set when $80 \%$ of data was used to develop protein-specific models by Gray et $a l .{ }^{8}$ is given in the table for Envision. For Deep2Full the Random85\% scan results are given. The prediction quality for ECNet was extracted from Figure 3 of Luo et al. ${ }^{9}$

\begin{tabular}{c|ccc|cc}
\hline \multirow{2}{*}{ Protein } & \multicolumn{3}{|c|}{ Pearson correlation } & \multicolumn{2}{c}{ Spearman Correlation } \\
\cline { 2 - 6 } & Deep2Full & Envision & Our model & ECNet & Our model \\
\hline$\beta$-lactamase & 0.89 & 0.85 & 0.91 & 0.87 & 0.90 \\
APH(3')-II & 0.78 & 0.84 & 0.77 & 0.75 & 0.76 \\
Hsp90 & 0.85 & 0.76 & 0.86 & 0.73 & 0.69 \\
MAPK1 & 0.77 & - & 0.79 & 0.47 & 0.76 \\
UBE2I & 0.67 & - & 0.72 & 0.72 & 0.72 \\
TPK1 & 0.42 & - & 0.34 & 0.31 & 0.34 \\
Bg13 & - & - & 0.75 & 0.74 & 0.75 \\
LGK & - & - & 0.52 & 0.44 & 0.44 \\
\hline
\end{tabular}


Table S5. Comparison of prediction quality of our XGBoost models with that of methods which do not require mutational effect scores of protein variants for generating the model. For DeepSequence ${ }^{10}$ and EVmutation ${ }^{11}$ the Spearman correlations reported in the supplementary information of Riesselman et al. ${ }^{10}$ are given. *Envision predictions for these proteins are from Leave-One-Protein-Out analysis (Supplementary Figure 8) of Gray et al. ${ }^{8}$.

\begin{tabular}{cccccc}
\hline & \multicolumn{5}{c}{ Spearman correlation } \\
\cline { 2 - 6 } Protein & $\begin{array}{c}\text { Deep- } \\
\text { Sequence }\end{array}$ & EVmutation & SNAP2 & Envision & Our model \\
\hline B-lactamase & 0.78 & 0.72 & 0.71 & $0.74^{*}$ & 0.90 \\
APH(3')-II & 0.59 & 0.54 & 0.49 & $0.64^{*}$ & 0.76 \\
Hsp90 & 0.53 & 0.49 & 0.43 & $0.31^{*}$ & 0.69 \\
MAPK1 & -0.24 & -0.25 & 0.30 & -0.44 & 0.76 \\
UBE2I & 0.55 & 0.51 & -0.51 & 0.09 & 0.72 \\
TPK1 & 0.26 & 0.25 & -0.22 & 0.27 & 0.34 \\
Bgl3 & 0.67 & 0.63 & 0.64 & - & 0.75 \\
LGK & 0.23 & 0.22 & 0.22 & - & 0.44 \\
\hline
\end{tabular}


Table S6. Different protein descriptors that were analyzed to check for the possible correlation with variations in the individual feature contributions to fitness across proteins.

\begin{tabular}{cccccccc}
\hline Protein & $\begin{array}{c}\text { number } \\
\text { of amino } \\
\text { acids }\end{array}$ & $\begin{array}{c}\text { number } \\
\text { of } \\
\text { domains }\end{array}$ & domain & $\begin{array}{c}\% \text { of } \\
\text { residues } \\
\text { in helices }\end{array}$ & $\begin{array}{c}\% \text { of } \\
\text { residues } \\
\text { in beta- } \\
\text { sheet }\end{array}$ & $\begin{array}{c}\% \text { of } \\
\text { charged } \\
\text { and polar } \\
\text { residues }\end{array}$ & $\begin{array}{c}\% \text { of hy- } \\
\text { drophilic } \\
\text { surface } \\
\text { area }\end{array}$ \\
\hline $\begin{array}{c}\beta- \\
\text { lactamase }\end{array}$ & 286 & 1 & 1 & 46 & 17 & 45 & 77 \\
APH(3')-II & 264 & 1 & 1 & 39 & 18 & 43 & 65 \\
Hsp90 & 709 & 3 & $\begin{array}{c}\text { ATPase } \\
\text { binding } \\
\text { domain }\end{array}$ & 32 & 16 & 55 & 72 \\
MAPK1 & 360 & 1 & 1 & 38 & 13 & 47 & 68 \\
UBE2I & 159 & 1 & 1 & 35 & 19 & 46 & 67 \\
TPK1 & 243 & 2 & both & 26 & 30 & 47 & 67 \\
Bg13 & 500 & 1 & 1 & 43 & 16 & 43 & 73 \\
LGK & 447 & 1 & 1 & 42 & 21 & 46 & 72 \\
\hline
\end{tabular}

\section{References}

[1] M. A. Stiffler, D. R. Hekstra, and R. Ranganathan, "Evolvability as a function of purifying selection in TEM-1 beta-lactamase," Cell, vol. 160, no. 5, pp. 882-892, 2015.

[2] A. Melnikov, P. Rogov, L. Wang, A. Gnirke, and T. S. Mikkelsen, "Comprehensive mutational scanning of a kinase in vivo reveals substrate-dependent fitness landscapes," Nucleic Acids Res., vol. 42, no. 14, p. e112, 2014.

[3] P. Mishra, J. M. Flynn, T. N. Starr, and D. N. A. Bolon, "Systematic mutant analyses elucidate general and client-specific aspects of Hsp90 function," Cell Rep., vol. 15, no. 3, pp. 588-598, 2016.

[4] L. Brenan, A. Andreev, O. Cohen, S. Pantel, A. Kamburov, D. Cacchiarelli, N. S. Persky, C. Zhu, M. Bagul, E. M. Goetz, A. B. Burgin, L. A. Garraway, G. Getz, T. S. Mikkelsen, F. Piccioni, D. E. Root, and C. M. Johannessen, "Phenotypic characterization of a comprehensive set of MAPK1/ERK2 missense mutants," Cell Rep., vol. 17, pp. 1171-1183, OCT 182016.

[5] J. Weile, S. Sun, A. G. Cote, J. Knapp, M. Verby, J. C. Mellor, Y. Wu, C. Pons, C. Wong, N. van Lieshout, F. Yang, M. Tasan, G. Tan, S. Yang, D. M. Fowler, R. Nussbaum, J. D. Bloom, 
M. Vidal, D. E. Hill, P. Aloy, and F. P. Roth, “A framework for exhaustively mapping functional missense variants," Mol. Syst. Biol., vol. 13, no. 12, 2017.

[6] P. A. Romero, T. M. Tran, and A. R. Abate, "Dissecting enzyme function with microfluidicbased deep mutational scanning," Proc. Natl. Acad. Sci. U. S. A., vol. 112, no. 23, pp. 71597164, 2015.

[7] J. R. Klesmith, J.-P. Bacik, R. Michalczyk, and T. A. Whitehead, "Comprehensive sequenceflux mapping of a levoglucosan utilization pathway in e. coli," ACS Synth. Biol., vol. 4, no. 11, pp. 1235-1243, 2015.

[8] V. E. Gray, R. J. Hause, J. Luebeck, J. Shendure, and D. M. Fowler, "Quantitative missense variant effect prediction using large-scale mutagenesis data," Cell Syst., vol. 6, no. 1, pp. 116124, 2018.

[9] Y. Luo, L. Vo, H. Ding, Y. Su, Y. Liu, W. W. Qian, H. Zhao, and J. Peng, "Evolutionary contextintegrated deep sequence modeling for protein engineering," bioRxiv, 2020.

[10] A. J. Riesselman, J. B. Ingraham, and D. S. Marks, "Deep generative models of genetic variation capture the effects of mutations," Nat. Methods, vol. 15, no. 10, pp. 816-822, 2018.

[11] T. A. Hopf, J. B. Ingraham, F. J. Poelwijk, C. P. I. Scharfe, M. Springer, C. Sander, and D. S. Marks, "Mutation effects predicted from sequence co-variation," Nat. Biotechnol., vol. 35, no. 2, pp. 128-135, 2017. 